JOURNAL OF INTEGRAL EQUATIONS

AND APPLICATIONS

Volume 5, Number 1, Winter 1993

\title{
THE $h-p$-VERSION \\ OF SPLINE APPROXIMATION METHODS FOR MELLIN CONVOLUTION EQUATIONS
}

\author{
J. ELSCHNER
}

\begin{abstract}
We consider the numerical solution of Mellin convolution equations on an interval by the $h$ - $p$-version of spline approximation methods. Using a geometric mesh refinement towards the singularity of the integral equation, we prove stability and exponential convergence in the $L_{q}$ norm, $1 \leq q \leq \infty$, for Galerkin, collocation and Nyström methods based on piecewise polynomials.
\end{abstract}

1. Introduction. We consider the approximate solution of the one-dimensional Mellin convolution equation

$$
u(x)-\int_{0}^{1} \kappa(x / y) u(y) y^{-1} d y=f(x), \quad x \in I:=(0,1),
$$

where $f$ and $\kappa$ are given functions and $u$ is the unknown function. Such integral equations having a fixed singularity at the point $x=0$ arise in a variety of applications; for example, they occur when boundary integral methods are applied to potential problems in plane regions with corners or to crack problems in linear elasticity (see $[4, \mathbf{1 3}]$ and the references therein). Note that the integral operator in (1.1) is not compact so that standard theories for the numerical analysis of second kind Fredholm integral equations cannot be applied. Nevertheless, using graded meshes and modified spline spaces, results on stability and optimal convergence orders of Galerkin, collocation and quadrature methods for Equation (1.1) which are based on piecewise polynomial basis functions have been obtained in $[\mathbf{4}, \mathbf{6}, \mathbf{7}, \mathbf{9}]$. These papers apply the technique of the traditional $h$-version of spline approximation methods where accuracy is achieved by decreasing the mesh size $h$, while keeping the degree $p$ of piecewise polynomials fixed.

In the present paper we study the $h$-p-version of those approximation methods which is obtained if one simultaneously refines the mesh and

Received by the editors on August 4, 1992.

Copyright (C)1993 Rocky Mountain Mathematics Consortium 
increases the degree of the splines. For the finite element method on a geometric mesh applied to elliptic boundary value problems in planar polygonal domains, it has been shown in [10] that, if the given data are piecewise analytic, the $h-p$-version has an exponential rate of convergence with respect to the number of degrees of freedom, whereas the $h$-version has only a polynomial rate. The state of the art of the $p$ and $h$ - $p$-versions of the finite element method is described in [3]. Only recently the $h$ - $p$-version has been introduced into boundary element methods $[\mathbf{2}, \mathbf{1 5}]$. These papers analyze the $h$ - $p$-version of boundary element Galerkin methods with quasiuniform and geometric meshes for some strongly elliptic first-kind integral equations on a polygon; [2] establishes, in particular, the exponential rate of convergence when a geometric mesh refinement towards the vertices is used.

Our aim is to show corresponding results for spline Galerkin methods applied to the second-kind integral equation (1.1). Furthermore, the $h$-p-version of certain collocation and Nyström quadrature methods is discussed here for the first time. In Section 2 we recall some analytical properties of (1.1) in $L_{q}(I), 1 \leq q \leq \infty$, and in weighted Sobolev spaces. Following [10] we further introduce countable normed spaces $B_{q}^{\varrho}(I)$ of real-analytic functions on $(0,1]$ which are adapted to the singularities of solutions to (1.1) at the origin. As one of our main results we prove that if the right-hand side $f$ of (1.1) belongs to $B_{q}^{\varrho}(I)$ and the kernel function $\kappa$ satisfies appropriate conditions then the solution $u$ is also an element of $B_{q}^{\varrho}(I)$.

In Section 3 we define the spline spaces $\mathcal{S}_{n, \sigma, \mu}, \sigma \in(0,1), \mu>0$, consisting of piecewise polynomials of degree $[\mu n]$ on the subintervals $\left[\sigma^{n-i}, \sigma^{n-i-1}\right], i=1, \ldots, n-1$, of the geometric mesh. (Here and in the following $[a]$ denotes the integral part of $a$.) On the first subinterval $\left[0, \sigma^{n-1}\right]$ the splines are assumed to be zero; this corresponds to the simplest modification of the usual spline spaces in order to deal with the singularity of the integral equation (1.1) (cf. $[\mathbf{4}, \mathbf{6}, \mathbf{7}, \mathbf{9}])$. Following [10], we investigate the $L_{q}$ approximation of functions $u \in B_{q}^{\varrho}(I)$ by splines from $\mathcal{S}_{n, \sigma, \mu}$. The underlying approximation theory, however, is simpler than that of $[\mathbf{1 0}]$ and essentially relies on Jackson's theorem. Besides the uniform degree distribution, we also briefly discuss the case of spline spaces with linear degree distribution; cf. (3.4) for definition.

In Section 4 we prove stability and exponential convergence in the $L_{q}$ norm $(1 \leq q \leq \infty)$ of Galerkin and collocation methods with 
basis functions from $\mathcal{S}_{n, \sigma, \mu}$. In Section 5 we present the corresponding results for the Nyström method which can be regarded as the discrete iterated collocation method based on the spline spaces $\mathcal{S}_{n, \sigma, \mu}$. Using a somewhat different approach, it is possible to treat Galerkin and collocation methods with splines which are not required to be zero over the first interval $\left[0, \sigma^{n-1}\right]$; cf. $[7]$ in case of the $h$-version.

2. Smoothness of solutions. We first recall some facts about the solvability of Equation (1.1) in weighted Sobolev spaces; see [7]. Let $A$ be the Mellin convolution operator defined by

$$
A u(x):=(1-K) u(x), \quad K u(x):=\int_{0}^{1} \kappa(x / y) u(y) y^{-1} d y .
$$

For any interval $J$ and $1 \leq q \leq \infty, L_{q}(J)$ will denote the usual Lebesgue space on $J$ with norm

$$
\begin{gathered}
\left\|u ; L_{q}(J)\right\|=\left\{\int_{J}|u|^{q} d x\right\}^{1 / q}, \quad q<\infty ; \\
\left\|u ; L_{\infty}(J)\right\|=\underset{J}{\operatorname{ess} \sup }|u(x)| .
\end{gathered}
$$

For any integer $l \geq 1$, we further introduce the weighted Sobolev space

$$
L_{q, l}(I)=\left\{u \in L_{q}(I): x^{j} D^{j} u \in L_{q}(I), j=1, \ldots, l\right\}
$$

on the unit interval, which is equipped with the canonical norm

$$
\left\|u ; L_{q, l}(I)\right\|=\sum_{0 \leq j \leq l}\left\|x^{j} D^{j} u ; L_{q}(I)\right\|, D=d / d x .
$$

Let $\tilde{\kappa}$ be the Mellin transform of the kernel function of $K$ :

$$
\tilde{\kappa}(z)=\int_{0}^{\infty} x^{z-1} \kappa(x) d x .
$$

The function $a(z)=1-\tilde{\kappa}(z)$ is called the symbol of the Mellin convolution operator $A$. For fixed $q, 1 \leq q \leq \infty$, and $\varrho>0$, we make the following set of assumptions on $\kappa$ and $a$. 
(A1) $\int_{0}^{\infty} x^{1 / q-1}|\kappa(x)| d x<\infty ; a(z) \neq 0, \quad \operatorname{Re} z=1 / q$; $\{\arg a(1 / q+i \xi)\}_{-\infty}^{\infty}=0$.

$\left(\mathrm{A} 2^{\varrho}\right) \int_{0}^{\infty} x^{1 / q-1-\varrho}|\kappa(x)| d x<\infty ; a(z) \neq 0,1 / q-\varrho \leq \operatorname{Re} z<1 / q$.

Here $1 / q:=0$ if $q=\infty$, and $\{\arg \cdot\}_{-\infty}^{\infty}$ denotes the change in argument of $a(1 / q+i \xi)$ as $\xi$ runs from $-\infty$ to $\infty$.

Note that the $L_{q}$ operator norm of $K$ is bounded by the integral appearing in (A1), and then the second condition of (A1) is equivalent to the invertibility of $A$ on $L_{q}(I)$. Under the assumptions (A1) and $\left(\mathrm{A} 2^{\varrho}\right), u \in L_{q}(I)$ and $A u \in L_{q}^{\varrho}(I)$ imply that $u \in L_{q}^{\varrho}(I)$, where $L_{q}^{\varrho}(I)$ denotes the weighted space $x^{\varrho} L_{q}(I)$. Note that the supremum of the numbers $\varrho$ for which condition $\left(\mathrm{A} 2^{\varrho}\right)$ is satisfied reflects the principal term of the asymptotics of solutions to (1.1) at the origin; cf. [5].

The conditions (A1) are of course necessary for the stability in $L_{q}(I)$ of any projection method applied to (1.1). To obtain sufficient stability conditions, we usually have to require some additional smoothness of the kernel function:

$$
\int_{0}^{\infty} x^{1 / q-1}\left|x^{j} D^{j} \kappa(x)\right| d x<\infty, \quad j=0, \ldots, l .
$$

Condition $\left(\mathrm{A} 3_{l}\right)$ implies that $K$ is a bounded map of $L_{q}(I)$ into $L_{q, l}(I)$, and together with (A1) this ensures the invertibility of $A$ on $L_{q, l}(I)$.

To derive exponential convergence rates for our spline approximation methods, the framework of weighted Sobolev spaces does not suffice, and following $[\mathbf{1 0}]$ we therefore introduce appropriate countable normed spaces of real-analytic functions on $(0,1]$. For $1 \leq q \leq \infty$ and $\varrho \geq 0$, let

$$
\begin{aligned}
B_{q}^{\varrho}(I)=\left\{u \in C^{\infty}(0,1]\right. & : \exists d>0 \text { independent of } j \text { such that } \\
& \left.\left\|x^{j-\varrho} D^{j} u ; L_{q}(I)\right\| \leq d^{j+1} j !, j=0,1, \ldots\right\} .
\end{aligned}
$$

The functions in $B_{q}^{\varrho}(I)$ are characterized by different constants $d$. If we wish to emphasize the dependence of a function $u$ on $d$, we shall write $u \in B_{q, d}^{\varrho}(I)$.

In order to state our result on smoothness of solutions with respect to the scale $B_{q}^{\varrho}(I)$, we introduce the hypothesis

$$
\left.\kappa\right|_{I} \in B_{q}^{\varrho}(I)
$$


Theorem 2.1. Assume (A1) and (A4 $\left.{ }^{\circ}\right)$, and suppose in addition that $\left(\mathrm{A} 2^{\varrho}\right)$ holds if $\varrho>0$. Then $u \in L_{q}(I)$ and $A u \in B_{q}^{\varrho}(I)$ imply $u \in B_{q}^{\varrho}(I)$.

To prove this, we need the following preliminary

Lemma 2.2. (i) If $u \in B_{q, d}^{o}(I)$, then

$$
\left\|(x D)^{j} u ; L_{q}(I)\right\| \leq(2 e d)^{j+1} j !, \quad j=0,1, \ldots .
$$

(ii) Conversely, if

$$
\left\|(x D)^{j} u ; L_{q}(I)\right\| \leq d^{j+1} j !, \quad j=0,1, \ldots,
$$

then $u \in B_{q, 2 e d}^{o}(I)$.

Proof. (i) Using the relation $x^{j} D^{j}=x D(x D-1) \cdots(x D-j+1)$, one obtains

$$
\begin{aligned}
\left\|x^{j} D^{j} u ; L_{q}(I)\right\| & \leq \sum_{i=0}^{j}\left(\begin{array}{c}
j \\
i
\end{array}\right) j^{i}\left\|(x D)^{j-i} u ; L_{q}(I)\right\| \\
& \leq \sum_{i=0}^{j}(j ! / i !) d^{j+1-i} j^{i} \leq(j+1) d^{j+1} j^{j} .
\end{aligned}
$$

Since by Stirling's formula,

$$
j^{j} \leq(2 \pi)^{-1 / 2} e^{j} j ! \leq e^{j} j !, \quad j=0,1, \ldots,
$$

we finally get

$$
\left\|x^{j} D^{j} u ; L_{q}(I)\right\| \leq(e d)^{j+1}(j+1) ! \leq(2 e d)^{j+1} j ! .
$$

(ii) Note that

$$
(x D)^{j}=\sum_{i=0}^{j} c_{i}^{j} x^{j-i} D^{j-i}
$$


with certain nonnegative numbers $c_{i}^{j}$. Applying the operator $x D$ to the last relation, we obtain

$$
c_{i}^{j+1}=c_{i}^{j}+(j+1-i) c_{i-1}^{j}, \quad c_{j+1}^{j}=c_{-1}^{j}:=0 .
$$

Now, by induction one can easily show that for any $i$ and $j$

$$
c_{i}^{j} \leq\left(\begin{array}{c}
j \\
i
\end{array}\right) j^{i} .
$$

Therefore we obtain the estimate

$$
\left\|(x D)^{j} u ; L_{q}(I)\right\| \leq \sum_{i=0}^{j}\left(\begin{array}{c}
j \\
i
\end{array}\right) j^{i}\left\|x^{j-i} D^{j-i} u ; L_{q}(I)\right\|
$$

which implies the assertion as before.

Proof of Theorem 2.1. Step 1. We first verify the assertion in the case $\varrho=0$. We have to show that, under the assumptions (A1) and $\left(\mathrm{A} 4^{o}\right), u \in L_{q}(I)$ and $A u \in B_{q}^{o}(I)$ imply $u \in B_{q}^{o}(I)$. Thus, by Lemma 2.2 , one may assume that for some $d \geq 1$

$$
\begin{gathered}
\left\|u ; L_{q}(I)\right\| \leq d, \\
\left\|(x D)^{j} A u ; L_{q}(I)\right\|+\left\|(x D)^{j} \kappa ; L_{q}(I)\right\| \leq d^{j+1} j !, \quad j=0,1, \ldots .
\end{gathered}
$$

Note that $u \in L_{q, j}(I)$ for any $j \geq 1$; cf. [7, Theorem 1.10]. Proceeding by induction on $j$, we suppose that for some $k \geq 1$ and $d_{1} \geq d$

$$
\left\|(x D)^{j} u ; L_{q}(I)\right\| \leq d_{1}^{j+1} j !, \quad j<k .
$$

To show (2.4) for $j=k$, we start with the relation

$$
x D K u(x)=K x D u(x)-\kappa(x) u(1), \quad u \in L_{q, 1}(I)
$$

which follows by applying the operator $x D$ to the integral

$$
K u(x)=\int_{0}^{1} \kappa(x / y) u(y) y^{-1} d y=\int_{x}^{\infty} \kappa(z) u(x / z) z^{-1} d z .
$$


By iteration, (2.5) yields for any $k \geq 1$

$$
(x D)^{k} A u=A(x D)^{k} u+\sum_{j<k}(x D)^{k-1-j} \kappa(x)(x D)^{j} u(1), \quad u \in L_{q, k}(I) .
$$

Applying Sobolev's embedding theorem for the interval $[1 / 2,1]$, one has the estimate

$$
\left|(x D)^{k-1} u(1)\right| \leq \varepsilon\left\|(x D)^{k} u ; L_{q}(I)\right\|+c(\varepsilon)||(x D)^{k-1} u ; L_{q}(I) \|
$$

for any $\varepsilon>0$ and $k \geq 1$. Together with (2.3) and (2.6), this implies

$$
\begin{aligned}
\left\|A(x D)^{k} u ; L_{q}(I)\right\| \leq & \left\|(x D)^{k} A u ; L_{q}(I)\right\| \\
& +d\left\{\varepsilon\left\|(x D)^{k} u ; L_{q}(I)\right\|+c(\varepsilon)\left\|(x D)^{k-1} u ; L_{q}(I)\right\|\right\} \\
& +\sum_{j \leq k-2} d^{k-j}(k-1-j) !\left\{\left\|(x D)^{j+1} u ; L_{q}(I)\right\|\right. \\
& \left.+c(1)\left\|(x D)^{j} u ; L_{q}(I)\right\|\right\} .
\end{aligned}
$$

Choosing $\varepsilon$ sufficiently small and using (2.3), (2.4) and the invertibility of $A$ in $L_{q}(I)$, we obtain from the last inequality

$$
\left\|(x D)^{k} u ; L_{q}(I)\right\| \leq c d^{k+1} k !+\sum_{j \leq k-2} c d_{1}^{j+2}(j+1) ! d^{k-j}(k-j-1) !,
$$

where $c \geq 1$ does not depend on $k$. Using the estimates

$$
(j+1) !(k-j-1) ! \leq(k-1) !
$$

and selecting $d_{1}=2 c d^{2}$, we finally have

$$
\left\|(x D)^{k} u ; L_{q}(I)\right\| \leq c d^{k+1} k !+\sum_{j \leq k-2} c d_{1}^{j+2} d^{k-j}(k-1) ! \leq d_{1}^{k+1} k ! .
$$

This shows (2.4) for $j=k$ and completes the proof of the assertion in view of Lemma 2.2 .

Step 2. Passing to the Mellin convolution operator $A_{\varrho}=x^{-\varrho} A x^{\varrho}$ with kernel function $x^{-\varrho} \kappa$, the assertion in the general case may be reduced to the situation considered in Step 1. Indeed, using the relations $(x D)^{j} x^{-\varrho} \kappa=x^{-\varrho}(x D-\rho)^{j} \kappa$ and the considerations in the 
proof of Lemma 2.2, $\kappa \in B_{q}^{\varrho}(I)$ implies that $x^{-\varrho} \kappa \in B_{q}^{o}(I)$. Further, by assumption, $A_{\varrho}$ is invertible on $L_{q}(I)$ while $A$ is invertible on $L_{q}(I)$ and $L_{q}^{\varrho}(I)$. Thus it suffices to verify that $v=x^{-\varrho} u \in L_{q}(I)$ and $A_{\varrho} v=x^{-\varrho} A u \in B_{q}^{o}(I)$ imply $v \in B_{q}^{o}(I)$, which was already done in Step 1.

Remark 2.3. Another analyticity assumption on $\kappa$, which is stronger than $\left(\mathrm{A} 4^{\varrho}\right)$ but usually satisfied in practice, is the following condition

$$
\int_{0}^{\infty} x^{1 / q-1-\varrho}\left|x^{j} D^{j} \kappa(x)\right| d x \leq d^{j+1} j !, \quad j=0,1, \ldots
$$

This condition implies that

$$
K\left(L_{q}^{\varrho}(I)\right) \subset B_{q}^{\varrho}(I) .
$$

Indeed, for the Mellin convolution operator $x^{j-\varrho} D^{j} K x^{\varrho}$ with kernel function $x^{j-\varrho} D^{j} \kappa$, one then obtains the estimate

$$
\begin{aligned}
\left\|x^{j-\varrho} D^{j} K u ; L_{q}(I)\right\| & =\left\|x^{j-\varrho} D^{j} K x^{\varrho}\left(x^{-\varrho} u\right) ; L_{q}(I)\right\| \\
& \leq\left(\int_{0}^{\infty} x^{1 / q-1-\varrho}\left|x^{j} D^{j} \kappa(x)\right| d x\right)\left\|x^{-\varrho} u ; L_{q}(I)\right\| \\
& \leq c d^{j+1} j !, \quad j=0,1, \ldots .
\end{aligned}
$$

Notice that (2.7) together with the invertibility of $A$ on $L_{q}(I)$ and $L_{q}^{\varrho}(I)$ yields immediately the assertion of Theorem 2.1 if one replaces condition $\left(\mathrm{A} 4^{\varrho}\right)$ by $\left(\mathrm{A} 5^{\varrho}\right)$ there. Moreover, the fact that $\left(\mathrm{A} 5^{\varrho}\right)$ implies $\left(\mathrm{A} 4^{\varrho}\right)$ can now easily be verified if we choose in $(2.5)$ a smooth function $u$, vanishing in a neighborhood of $x=0$ and such that $u(1) \neq 0$, and observe that $x D K u-K x D u \in B_{q}^{\varrho}(I)$.

Example 2.4. Consider the operator (2.1) with kernel function $\kappa(x)=-\chi_{I}(x) x^{\beta}, \beta>0$, where $\chi_{I}$ denotes the characteristic function of the interval $I$ (i.e., $\chi_{I}(x)=1$ if $x \in I$ and $\chi_{I}(x)=0$ otherwise). Then the symbol of $A$ takes the form $a(z)=1+(\beta+z)^{-1}$, and the conditions (A1), $\left(\mathrm{A} 2^{\varrho}\right)$ and $\left(\mathrm{A} 4^{\varrho}\right)$ are satisfied for $1 \leq q \leq \infty$ and $0 \leq \varrho<\beta+1 / q$.

Example 2.5. Let $\kappa(x)=1 / \pi\left(1+x^{2}\right)$. The corresponding equation (1.1) appears as a local model when the single layer potential is 
used to solve the exterior Neumann problem for Laplace's equation and the plane domain has a corner with right angle. Then $a(z)=$ $1-1 / 2 \sin (\pi z / 2)$, and the assumptions (A1), $\left(\mathrm{A} 2^{\varrho}\right),\left(\mathrm{A} 3_{l}\right)$ and $\left(\mathrm{A} 5^{\varrho}\right)$ are fulfilled if $1 \leq q<3,0 \leq \varrho<1 / q-1 / 3$ and $l=1,2, \ldots$.

3. Auxiliary results from approximation theory. For $1 \leq q \leq$ $\infty$ and $k=0,1, \ldots$, let $W_{q}^{k}(I)$ denote the usual Sobolev space of order $k$ on the unit interval, where $W_{q}^{o}(I)=L_{q}(I)$. By $E_{m}\left(u ; W_{q}^{k}\right)$ we denote the error in the best approximation of the function $u$ by polynomials of degree $\leq m$ with respect to the norm in $W_{q}^{k}(I)$ :

$$
E_{m}\left(u ; W_{q}^{k}\right)=\inf \left\{\left\|u-\varphi ; W_{q}^{k}(I)\right\|: \varphi \in \mathbf{P}_{m}\right\} .
$$

The following proposition, being a simple consequence of Jackson's theorem, is the key to the derivation of exponential convergence rates for our spline approximation methods.

Proposition 3.1. We have

(i) $E_{m}\left(u ; L_{q}\right) \leq c^{k}(1+m)^{-k}\left\|D^{k} u ; L_{q}(I)\right\|, u \in W_{q}^{k}(I), m+1 \geq k$;

(ii) $E_{m}\left(u ; W_{q}^{1}\right) \leq c^{k+1}(1+m)^{-k}\left\|D^{k+1} u ; L_{q}(I)\right\|, u \in W_{q}^{k+1}(I)$, $m \geq k$,

where $c$ does not depend on $u, m$ and $k$.

Proof. If $k=0$, then the result is trivially true. Let $k \geq 1$.

(i) By Jackson's theorem

$$
E_{m}\left(u ; L_{q}\right) \leq c(1+m)^{-1}\left\|D u ; L_{q}(I)\right\|,
$$

and applying successively the relations

$$
E_{m-j}\left(D^{j} u ; L_{q}\right) \leq c(1+m-j)^{-1} E_{m-j-1}\left(D^{j+1} u ; L_{q}\right), \quad j=0,1, \ldots
$$

and an elementary inequality, one obtains (see [12, Chapter 1.6])

$$
E_{m}\left(u ; L_{q}\right) \leq c^{k}\left(k^{k} / k !\right)(1+m)^{-k}\left\|D^{k} u ; L_{q}(I)\right\| .
$$

Together with Stirling's formula (cf. (2.2)), this yields the result. 
(ii) By (i) there exists a polynomial $\psi \in \mathbf{P}_{m-1}$ such that

$$
\left\|D u-\psi ; L_{q}(I)\right\| \leq c^{k} m^{-k}\left\|D^{k+1} u ; L_{q}(I)\right\|, \quad m \geq k \geq 1,
$$

and setting $\varphi(x)=\int_{0}^{x} \psi(t) d t+u(0)$, the last estimate implies

$$
\left\|u-\varphi ; W_{q}^{1}(I)\right\| \leq c_{1}\left\|D(u-\varphi) ; L_{q}(I)\right\| \leq c_{1} c^{k} m^{-k}\left\|D^{k+1} u ; L_{q}(I)\right\|,
$$

where $c, c_{1}$ only depend on $q$.

Let $\mathcal{R}_{m}$ be the orthogonal projection of $L_{2}(I)$ onto $\mathbf{P}_{m}$. To investigate the $L_{q}$ convergence of spline Galerkin methods, we need the following simultaneous approximation result.

Proposition 3.2. For any $u \in W_{q}^{k+1}(I), 1 \leq q \leq \infty$ and $m \geq k$,

$$
\left\|\left(1-\mathcal{R}_{m}\right) u ; L_{q}(I)\right\| \leq c^{k+1}(1+m)^{-k}|| D^{k+1} u ; L_{q}(I) \|,
$$

where $c$ is independent of $u, m$ and $k$.

Proof. 1. Let $1 \leq q \leq 2$. Then, for any $\varphi \in \mathbf{P}_{m}$, the estimate

$$
\begin{aligned}
\left\|\left(1-\mathcal{R}_{m}\right) u ; L_{q}(I)\right\| & \leq\left\|\left(1-\mathcal{R}_{m}\right) u ; L_{2}(I)\right\| \\
& \leq\left\|u-\varphi ; L_{2}(I)\right\| \leq c_{1}\left\|u-\varphi ; W_{q}^{1}(I)\right\|
\end{aligned}
$$

holds, where $c_{1}$ only depends on $q$. Here we have used Sobolev's embedding theorem. Now it suffices to apply Proposition 3.1 (ii).

2. If $2 \leq q \leq \infty$, we have for any $\varphi \in \mathbf{P}_{m}$

$$
\begin{aligned}
\left\|\left(1-\mathcal{R}_{m}\right) u ; L_{q}(I)\right\| & \leq\left\|u-\varphi ; L_{q}(I)\right\|+\left\|\mathcal{R}_{m}(u-\varphi) ; L_{q}(I)\right\| \\
& \leq\left\|u-\varphi ; L_{q}(I)\right\|+c_{1}(1+m)^{1-2 / q}\left\|u-\varphi ; L_{q}(I)\right\| \\
& \leq\left(1+c_{1}\right)(1+m)^{1-2 / q}\left\|u-\varphi ; L_{q}(I)\right\|,
\end{aligned}
$$

where $c_{1}$ is independent of $u, \varphi$ and $m$ and the second inequality is a consequence of [14, Theorem 3.3]. It remains to apply Proposition 3.1 (i). 
To the Chebyshev nodes on $I$

$$
\xi_{j}=\xi_{j}^{(m)}:=\frac{1}{2}\left\{1+\cos \frac{(2 j+1) \pi}{2(m+1)}\right\}, \quad j=0, \ldots, m,
$$

we now associate the corresponding Lagrange interpolatory projection $\mathcal{L}_{m}$ onto $\mathbf{P}_{m}$. The following result is needed in the convergence analysis of spline collocation methods and is the analogue of the preceding proposition.

Proposition 3.3. For any $u \in W_{q}^{k+1}(I), 1 \leq q \leq \infty$ and $m \geq k$,

$$
\left\|\left(1-\mathcal{L}_{m}\right) u ; L_{q}(I)\right\| \leq c^{k+1}(1+m)^{-k}\left\|D^{k+1} u ; L_{q}(I)\right\|,
$$

where $c$ does not depend on $u, m$ and $k$.

Proof. 1. Let $q<\infty$. By a theorem of Erdös and Feldheim (cf. [16, Chapter 14.3]), we have

$$
\left\|\mathcal{L}_{m} u ; L_{q}(I)\right\| \leq c(q)\left\|u ; L_{\infty}(I)\right\|
$$

for all $m \in \mathbf{N}$ and all continuous functions $u$ on [0,1]. Utilizing Sobolev's embedding theorem, we thus obtain for any $\varphi \in \mathbf{P}_{m}$

$$
\begin{aligned}
\left\|\left(1-\mathcal{L}_{m}\right) u ; L_{q}(I)\right\| & \leq\left\|u-\varphi ; L_{q}(I)\right\|+\left\|\mathcal{L}_{m}(u-\varphi) ; L_{q}(I)\right\| \\
& \leq(1+c(q))\left\|u-\varphi ; L_{\infty}(I)\right\| \leq c_{1}(q)\left\|u-\varphi ; W_{q}^{1}(I)\right\|
\end{aligned}
$$

and it suffices to apply Proposition 3.1 (ii).

2. Let $q=\infty$. By a theorem of Bernstein (see [13, Chapter 3.3.1]), the $L_{\infty}$ operator norm of $\mathcal{L}_{m}$ is bounded by $c_{1}(1+\log (1+m))$. Now we obtain as before

$$
\left\|\left(1-\mathcal{L}_{m}\right) u ; L_{\infty}(I)\right\| \leq c_{1}(1+\log (1+m))\left\|u-\varphi ; L_{\infty}(I)\right\|
$$

for any $\varphi \in \mathbf{P}_{m}$, and Proposition 3.1 (i) completes the proof.

We now introduce the spaces of piecewise polynomials on geometric meshes occurring in the $h$ - $p$-version of spline approximation methods. 
For any $n \in \mathbf{N}$ and fixed $\sigma \in(0,1)$, we first define the geometric mesh $\Delta_{n}=\Delta_{n, \sigma}=\left\{x_{i}: 0 \leq i \leq n\right\}$ on $I$, where

$$
x_{i}=x_{i}^{(n)}=\sigma^{n-i}, \quad i=1, \ldots, n ; x_{o}=0 .
$$

Let $I_{i}=\left(x_{i-1}, x_{i}\right), h_{i}=x_{i}-x_{i-1}$, where the upper index $n$ is omitted for convenience. To the mesh $\Delta_{n, \sigma}$ and a fixed parameter $\mu>0$, we then associate the spline space

$$
\mathcal{S}_{n}=\mathcal{S}_{n, \sigma, \mu}:=\left\{u \in L_{\infty}(I):\left.u\right|_{I_{1}}=0,\left.u\right|_{I_{i}} \in \mathbf{P}_{[\mu n]}, i \geq 2\right\}
$$

which corresponds to a uniform degree distribution on the subintervals of $\Delta_{n}$. Sometimes we also consider the case of linear degree distribution

$$
\mathcal{S}_{n, \sigma, \mu}^{l i n}:=\left\{u \in L_{\infty}(I):\left.u\right|_{I_{1}}=0,\left.u\right|_{I_{i}} \in \mathbf{P}_{[\mu i]}, i \geq 2\right\} .
$$

The following theorem is the crucial approximation property of $\mathcal{S}_{n}$; for $q=2$ it is of course a special case of the two-dimensional results obtained in [10]. Let $P_{n}$ denote the orthogonal projection of $L_{2}(I)$ onto $\mathcal{S}_{n, \sigma, \mu}$.

Theorem 3.4. Let $u \in B_{q}^{\varrho}(I), 1 \leq q \leq \infty$ and $\varrho>0$. Then

$$
\left\|\left(1-P_{n}\right) u ; L_{q}(I)\right\| \leq c e^{-b n},
$$

where the constants $c$ and $b$ do not depend on $n$.

Proof. Step 1. On the first subinterval $\left(0, x_{1}\right)$ of the mesh $\Delta_{n}$, we have

$$
\left\|u ; L_{q}\left(I_{1}\right)\right\| \leq \sigma^{(n-1) \varrho}\left\|x^{-\varrho} u ; L_{q}\left(I_{1}\right)\right\| \leq c_{1} \sigma^{n \varrho} .
$$

Here and in the sequel $c, c_{1}, \ldots$ denote various constants not depending on $n$ and $\mu$.

Step 2. Next we prove the estimate

$$
\left\|u-P_{n} u ; L_{q}\left(x_{1}, 1\right)\right\| \leq c_{1} \sigma^{n \mu / c}
$$

in the case $q=2$. In order to do so, we choose a polynomial $\varphi_{i}$ on each subinterval $I_{i}, i \geq 2$, such that according to Proposition 3.1 (i) 
(with $m=[\mu n]$ and $k=[\nu n], 0<\nu<\mu$ ) and the scaling argument, the estimate

$$
\begin{aligned}
\left\|u-\varphi_{i} ; L_{2}\left(I_{i}\right)\right\| & \leq\left\{c_{2} h_{i} /(1+[\mu n])\right\}^{[\nu n]}\left\|D^{[\nu n]} u ; L_{2}\left(I_{i}\right)\right\| \\
& \leq\left\{c_{2} h_{i} / x_{i-1}(1+[\mu n])\right\}^{[\nu n]} x_{i}^{\varrho}\left\|x^{[\nu n]-\varrho} D^{[\nu n]} u ; L_{2}\left(I_{i}\right)\right\|
\end{aligned}
$$

holds. Here $c_{2}$ is also independent of $i$ and $\nu$, and $\nu$ will be chosen sufficiently small later on. Since $h_{i} / x_{i-1}=(1-\sigma) / \sigma$ (cf. (3.2)) and $u \in B_{2}^{\varrho}(I)$, the last expression in (3.8) can be bounded by

$$
\left\{c_{3} /(1+[\mu n])\right\}^{[\nu n]} x_{i}^{\varrho}[\nu n] ! .
$$

Therefore we obtain from (3.8)

$$
\begin{aligned}
\left\{\sum_{i \geq 2}\left\|u-\varphi_{i} ; L_{2}\left(I_{i}\right)\right\|^{2}\right\}^{1 / 2} & \leq c_{4}\left\{c_{3} /(1+[\mu n])\right\}^{[\nu n]}[v n] ! \\
& \leq c_{4}\left(c_{3} \frac{1+[\nu n]}{1+[\mu n]}\right)^{[\nu n]}
\end{aligned}
$$

since $[\nu n] !(1+[\nu n])^{-[\nu n]} \leq 1$. Choosing now $\nu=\mu / c$ with sufficiently large $c$ in (3.9), we get (3.7) for $q=2$. Indeed, if $c=2 c_{3} / \sigma$ and $\mu n / c \geq 1$, the last expression in (3.9) can be dominated by $c_{4} \sigma^{[\nu n]} \leq c_{1}^{\nu n}$, whereas for all indices $n$ with $\mu n / c<1$ it can be bounded by $c_{4} \leq c_{2} \sigma^{\nu n}$.

Step 3. We finally verify (3.7) for arbitrary $q$. Then (3.6) and (3.7) obviously imply estimate (3.5). Arguing as before, but applying Proposition 3.2 instead of Proposition 3.1 (i), we get for $i \geq 2$

$$
\left\|u-P_{n} u ; L_{q}\left(I_{i}\right)\right\| \leq c_{3}\left\{c_{3} /(1+[\mu n])\right\}^{[\nu n]} x_{i}^{\varrho}(1+[\nu n]) ! .
$$

By virtue of the obvious estimate $(1+[\nu n]) !(1+[\nu n])^{-[\nu n]} \leq 1$, this yields again

$$
\left\|u-P_{n} u ; L_{q}\left(x_{1}, 1\right)\right\| \leq c_{4}\left(c_{3} \frac{1+[\nu n]}{1+[\mu n]}\right)^{[\nu n]}
$$

hence the result. 
Remark 3.5. Let $N$ be the number of degrees of freedom (i.e., the dimension) of $\mathcal{S}_{n, \sigma, \mu}$. Then $N \leq \mu n^{2}$, i.e., $n \geq \sqrt{N / \mu}$, and (3.6) and (3.7) imply the estimate

$$
\left\|\left(1-P_{n}\right) u ; L_{q}(I)\right\| \leq c_{1}\left\{\sigma^{\varrho \sqrt{N / \mu}}+\sigma^{\sqrt{N \mu} / c}\right\} \leq c_{2} e^{-b \sqrt{N}},
$$

where $c, c_{1}$ and $c_{2}$ do not depend on $N$ and $\mu$, while $b$ is independent of $N$ but depends on $\mu$. Note that a somewhat more precise estimate for $q=2$ and a discussion of the optimal choice of $\mu$ and $\sigma$ can be found in $[\mathbf{1 0}]$. The starting point there is the more precise version of Proposition 3.1 (i)

$$
E_{m}\left(u ; L_{2}\right)^{2} \leq \frac{(m-k+1) !}{(m+k+1) !}\left\|D^{k} u ; L_{2}(I)\right\|^{2}, \quad m+1 \geq k
$$

which is shown by approximating $u$ by the Legendre polynomials.

Remark 3.6. If $P_{n}$ denotes the orthogonal projection of $L_{2}(I)$ onto $\mathcal{S}_{n, \sigma, \mu}^{\operatorname{lin}}$ (cf. (3.4)), then the assertion of Theorem 3.4 only holds, in general, if $\mu$ is sufficiently large. Indeed, instead of estimate (3.10), one obtains from Proposition 3.2 that

$$
\left\|u-P_{n} u ; L_{q}\left(I_{i}\right)\right\| \leq c_{3} x_{i}^{\varrho}\left\{c_{3} /(1+[\mu i])\right\}^{i}(1+i) !, \quad i \geq 2,
$$

which gives

$$
\left\|u-P_{n} u ; L_{q}\left(I_{i}\right)\right\| \leq c_{4}\left\{c_{3} \sigma^{-\varrho}(1+i) /(1+[\mu i])\right\}^{i} \sigma^{\varrho n} \leq c_{4} 2^{-i} \sigma^{\varrho n}
$$

if $\mu$ is large enough. These estimates yield

$$
\left\|u-P_{n} u ; L_{q}\left(x_{1}, 1\right)\right\| \leq c_{4} \sigma^{\varrho n}
$$

and consequently

$$
\left\|\left(1-P_{n}\right) u ; L_{q}(I)\right\| \leq c_{1} \sigma^{\varrho n} \leq c_{2} e^{-b \sqrt{N / \mu}},
$$

where $N=\operatorname{dim} \mathcal{S}_{n, \sigma, \mu}^{\text {lin }}$ and $c_{1}, c_{2}, b$ are independent of $n, N$ and $\mu$. This corresponds to the result in $[\mathbf{1 0}]$ for $q=2$. 
Finally, to the Chebyshev nodes (3.1) and the mesh $\Delta_{n, \sigma}$ we associate the collocation points

$$
x_{i j}=x_{i j}^{(n)}=x_{i-1}+\xi_{j} h_{i}, \quad(i, j) \in \mathcal{T},
$$

where $\mathcal{T}=\{(i, j): 2 \leq i \leq n, 0 \leq j \leq[\mu n]\}$. For any continuous function $u$ on $(0,1]$, define the interpolatory projection $Q_{n} u \in \mathcal{S}_{n, \sigma, \mu}$ by

$$
\left(Q_{n} u\right)\left(x_{i j}\right)=u\left(x_{i j}\right), \quad(i, j) \in \mathcal{T} .
$$

To prove exponential convergence of spline collocation methods, we need the following analogue of Theorem 3.4.

Theorem 3.7. If $u \in B_{q}^{\varrho}(I), 1 \leq q \leq \infty$ and $\varrho>0$, then

$$
\left\|\left(I-Q_{n}\right) u ; L_{q}(I)\right\| \leq c e^{-b n},
$$

where $c$ and $b$ are independent of $n$.

Proof. This follows as in Step 3 of the proof of Theorem 3.4, using of course Proposition 3.3 in place of Proposition 3.2.

4. Stability and exponential convergence of Galerkin and collocation methods. Let $A=1-K$ be the Mellin convolution operator defined in (2.1). For the approximate solution of Equation (1.1), we first consider the $h$-p-version of spline Galerkin methods, namely the Galerkin method with splines from $\mathcal{S}_{n}=\mathcal{S}_{n, \sigma, \mu}$. The Galerkin solution $u_{n} \in \mathcal{S}_{n}$ is defined by

$$
P_{n} A u_{n}=u_{n}-P_{n} K u_{n}=P_{n} f .
$$

Theorem 4.1. (i) Assume (A1), and suppose additionally that condition $\left(\mathrm{A}_{2}\right)$ holds if $q \neq 2$. Then the Galerkin method (4.1) is stable in $L_{q}(I)$, i.e.,

$$
\left\|P_{n} A u_{n} ; L_{q}(I)\right\| \geq c\left\|u_{n} ; L_{q}(I)\right\|, \quad u_{n} \in \mathcal{S}_{n}, n \geq n_{0},
$$


where $n_{0}$ is large enough and $c$ does not depend on $u_{n}$ and $n$.

(ii) Under the conditions of (i) and the assumptions $f \in B_{q}^{\varrho}(I)$, $\varrho>0,\left(\mathrm{~A} 2^{\varrho}\right)$ and $\left(\mathrm{A} 4^{\varrho}\right)$, the Galerkin solution $u_{n}$ of $(4.1)$ converges exponentially to the exact solution $u$ of (1.1) in $L_{q}(I)$, i.e.,

$$
\left\|u-u_{n} ; L_{q}(I)\right\| \leq c e^{-b \sqrt{N}},
$$

where $N$ is the number of degrees of freedom, $c$ and $b$ are some constants not depending on $N$ (if $\mu$ is fixed).

Note that the Galerkin method (4.1) is stable in $L_{2}(I)$ under the minimal (invertibility) assumptions on $A$, whereas in our approach the stability in $L_{q}(I), q \neq 2$, requires some additional smoothness of the kernel function. The proof of Theorem 4.1 (i) is based on the stability of the finite section method for (1.1). Consider the truncation operators

$$
\pi_{n} u=\chi_{\left(x_{1}, 1\right)} u, \quad x_{1}=x_{1}^{(n)}=\sigma^{n-1} .
$$

Lemma 4.2. If assumption (A1) is satisfied, then

$$
\left\|\pi_{n} A \pi_{n} u ; L_{q}(I)\right\| \geq c\left\|\pi_{n} u ; L_{q}(I)\right\|, \quad u \in L_{q}(I), n \geq n_{0},
$$

where $n_{0}$ is sufficiently large and $c$ is independent of $u$ and $n$.

Proof. Consider the map $\Phi u(x)=e^{-x / q} u\left(e^{-x}\right)$ which is an isomorphism of $L_{q}(I)$ onto $L_{q}(0, \infty)$. Then $\Phi(1-K) \Phi^{-1}$ becomes the WienerHopf integral operator

$$
1-W, W u(x):=\int_{0}^{\infty} w(x-y) u(y) d y,
$$

with kernel function $w(x)=e^{-x / q} \kappa\left(e^{-x}\right) \in L_{1}(-\infty, \infty)$. Now (4.5) is a consequence of the stability of the finite section method for WienerHopf equations; see [8] for $q<\infty$ and [1] for $q=\infty$, or [13].

Proof of Theorem 4.1. (i) For any $u_{n} \in \mathcal{S}_{n}$, the relation

$$
P_{n}(1-K) u_{n}=\pi_{n}(1-K) \pi_{n} u_{n}+\pi_{n}\left(1-P_{n}\right) K u_{n}
$$


holds. Therefore, by virtue of (4.5), for the proof of estimate (4.2), it is sufficient to verify that

$$
\left\|\pi_{n}\left(1-P_{n}\right) K\right\|_{q} \rightarrow 0, \quad n \rightarrow \infty
$$

where $\|\cdot\|_{q}$ denotes the operator norm on $L_{q}(I)$. We first consider the case $q=2$.

Let $u \in L_{2}(I)$. In the sequel $c, c_{1}, \ldots$ denote various constants not depending on $u, n$ and $i$. Applying Proposition 3.1 (i) (with $k=1$ ) and the scaling argument, we obtain for $i \geq 2$

$$
\begin{aligned}
& \left\|\left(1-P_{n}\right) K u ; L_{2}\left(I_{i}\right)\right\| \leq c h_{i}(1+[\mu n])^{-1}\left\|D K u ; L_{2}\left(I_{i}\right)\right\| \\
& \leq c\left(h_{i} / x_{i-1}\right)(1+[\mu n])^{-1}\left\|x D K u ; L_{2}\left(I_{i}\right)\right\| \leq c_{1} n^{-1}\left\|x D K u ; L_{2}\left(I_{i}\right)\right\| .
\end{aligned}
$$

If we assume, in addition, that $K$ is a continuous map of $L_{2}(I)$ into $L_{2,1}(I)$, then (4.8) implies that

$$
\left\|\pi_{n}\left(1-P_{n}\right) K u ; L_{2}(I)\right\| \leq c_{1} n^{-1}|| x D K u ; L_{2}(I)\left\|\leq c_{2} n^{-1}\right\| u ; L_{2}(I) \|
$$

which proves (4.7) for $q=2$. Now we observe that, for any $\varepsilon>0$, $K$ may be approximated by a convolution operator $K_{\varepsilon}$ with kernel $\kappa_{\varepsilon}$ satisfying condition $\left(\mathrm{A} 3_{1}\right)$ such that $\left\|K-K_{\varepsilon}\right\|_{2} \leq \varepsilon$. Since the projections $P_{n}$ are, of course, uniformly bounded on $L_{2}(I)$, it suffices to verify the $L_{2}$ stability of the Galerkin method for $1-K_{\varepsilon}$ if $\varepsilon$ is sufficiently small.

Next we prove (4.7) for arbitrary $q$. Applying Proposition 3.2 (with $k=1$ ) and the scaling argument, we get for any $u \in L_{q}(I)$ and $i \geq 2$

$$
\begin{aligned}
\left\|\left(1-P_{n}\right) K u ; L_{q}\left(I_{i}\right)\right\| & \leq c h_{i}^{2}(1+[\mu n])^{-1}\left\|D^{2} K u ; L_{q}\left(I_{i}\right)\right\| \\
& \leq c\left(h_{i} / x_{i-1}\right)^{2}(1+[\mu n])^{-1}\left\|x^{2} D^{2} K u ; L_{q}\left(I_{i}\right)\right\| \\
& \leq c_{1} n^{-1}\left\|x^{2} D^{2} K u ; L_{q}\left(I_{i}\right)\right\| .
\end{aligned}
$$

Since $K$ is a bounded operator of $L_{q}(I)$ into $L_{q, 2}(I)$ by condition $\left(\mathrm{A} 3_{2}\right)$, it follows from the last estimate that

$$
\begin{aligned}
\left\|\pi_{n}\left(1-P_{n}\right) K u ; L_{q}(I)\right\| & \leq c_{1} n^{-1}\left\|x^{2} D^{2} K u ; L_{q}(I)\right\| \\
& \leq c_{2} n^{-1}\left\|u ; L_{q}(I)\right\|
\end{aligned}
$$

which completes the stability proof. 
(ii) By assumption and Theorem 2.1, the exact solution $u$ of (1.1) belongs to $B_{q}^{\varrho}(I)$. Therefore, in view of Theorem 3.4 and Remark 3.5, it is now sufficient to verify the estimate

$$
\left\|u-u_{n} ; L_{q}(I)\right\| \leq c\left\|\left(1-P_{n}\right) u ; L_{q}(I)\right\|, \quad n \geq n_{0} .
$$

Since $\left\|u-u_{n} ; L_{q}(I)\right\| \leq\left\|\left(1-P_{n}\right) u ; L_{q}(I)\right\|+\left\|P_{n} u-u_{n} ; L_{q}(I)\right\|$ and the stability estimate (4.2) implies

$$
\left\|P_{n} u-u_{n} ; L_{q}(I)\right\| \leq c\left\|P_{n} A\left(1-P_{n}\right) u ; L_{q}(I)\right\|, \quad n \geq n_{0},
$$

it remains to verify that the last expression is bounded by $c \|(1-$ $\left.P_{n}\right) u ; L_{q}(I) \|$. For $q=2$, this is obvious because of the uniform boundedness of $P_{n}$. In the general case, we have by (4.9)

$$
\begin{aligned}
\left\|P_{n} A\left(1-P_{n}\right) u ; L_{q}(I)\right\| \leq & \left\|\pi_{n} K\left(1-P_{n}\right) u ; L_{q}(I)\right\| \\
& +\left\|\pi_{n}\left(1-P_{n}\right) K\left(1-P_{n}\right) u ; L_{q}(I)\right\| \\
\leq & c\left\|\left(1-P_{n}\right) u ; L_{q}(I)\right\|
\end{aligned}
$$

which completes the proof.

We now consider the $h$-p-version of spline collocation methods. We seek an element $u_{n} \in \mathcal{S}_{n}$ such that

$$
Q_{n} A u_{n}=u_{n}-Q_{n} K u_{n}=Q_{n} f,
$$

where $Q_{n}$ denotes the interpolatory projection defined in (3.13). The following result is the analogue of Theorem 4.1 for the collocation method. Its proof follows the same line as there, using of course Proposition 3.3 and Theorem 3.7 instead of Proposition 3.2 and Theorem 3.4.

Theorem 4.3 (i) Assume (A1) and (A32). Then the collocation method (4.10) is stable in $L_{q}(I)$, i.e., estimate (4.2) holds with $P_{n}$ replaced by $Q_{n}$.

(ii) If, in addition, the assumptions $f \in B_{q}^{\varrho}(I), \varrho>0,\left(\mathrm{~A} 2^{\varrho}\right)$ and $\left(\mathrm{A} 4^{\circ}\right)$ are satisfied, then the collocation method converges exponentially in $L_{q}(I)$, i.e., estimate (4.3) holds with the collocation solution $u_{n}$ of (4.10). 
Remark 4.4. For the approximation methods (4.1) and (4.10) with basis functions from $\mathcal{S}_{n, \sigma, \mu}^{\text {lin }}$ (cf. (3.4)), the results of this section hold if $\mu$ is sufficiently large; compare Remark 3.6 and the proof of Theorem 4.1. To derive stability of those methods, one has to use, for example, the estimates

$$
\left\|\left(1-P_{n}\right) K u ; L_{2}\left(I_{i}\right)\right\| \leq c h_{i}(1+[\mu i])^{-1}\left\|D K u ; L_{2}\left(I_{i}\right)\right\|, \quad i \geq 2
$$

instead of (4.8).

Remark 4.5. In contrast to collocation methods based on piecewise polynomials of fixed degree (cf. $[4,7])$, for the $h$-p-version one does not have so much freedom in the choice of collocation points. If we take, for example, equidistant points $\xi_{j}=\xi_{j}^{(m)}=j / m, j=0, \ldots, m$, on the unit interval, then the $L_{\infty}$ operator norm of the corresponding Lagrange interpolatory projection $\mathcal{L}_{m}$ grows exponentially as $m \rightarrow \infty$ (cf., e.g., [11]) so that the results of Proposition 3.3 and Theorem 4.3 cannot be expected in this case. Selecting, however, $\xi_{j}^{(m)}$ as the GaussLegendre points on $I$, one has the estimate (see [16, Chapter 14.4])

$$
\left\|\mathcal{L}_{m} u ; L_{\infty}(I)\right\| \leq c \sqrt{m}\left\|u ; L_{\infty}(I)\right\|, \quad m=1,2, \ldots
$$

for any continuous function $u$ on $[0,1]$, and defining $Q_{n}$ again by (3.13), Theorem 4.3 holds with this choice of the collocation points $x_{i j}$. This only requires a slight modification of the corresponding proofs.

5. Nyström methods. We finally study stability and exponential convergence of a quadrature method which can be interpreted as the discrete iterated version of the collocation method (4.10). To define this method, consider the $(m+1)$-point interpolatory quadrature rule

$$
\int_{0}^{1} v d x \sim \sum_{j=0}^{m} \omega_{j}^{(m)} v\left(\xi_{j}^{(m)}\right)=\int_{0}^{1} \mathcal{L}_{m} v d x
$$

with weights $\omega_{j}=\omega_{j}^{(m)}, j=0, \ldots, m$ and the Chebyshev nodes $\xi_{j}=\xi_{j}^{(m)}$ introduced in (3.1). The following property which is a consequence of a theorem of Fejer (cf. [12, Chapter 3.6.2]) is of importance for our convergence analysis:

$$
\omega_{j}^{(m)} \geq 0 \quad \text { for all } j \text { and } m \text {. }
$$


Let $Q_{n}$ be the interpolatory projection onto $\mathcal{S}_{n, \sigma, \mu}$ defined in (3.13) via the collocation points $x_{i j},(i, j) \in \mathcal{T}$. Then the composite quadrature rule obtained by shifting (5.1) (with $m=[\mu n]$ ) to each subinterval $I_{i}$, $i \geq 2$, of the geometric mesh $\Delta_{n, \sigma}$ and summing over $i \geq 2$ is

$$
\int_{0}^{1} v d x \sim \sum_{(i, j) \in \mathcal{T}} \omega_{j} v\left(x_{i j}\right) h_{i}=\int_{0}^{1} Q_{n} v d x .
$$

Using (5.3) we approximate the integral operator $K$ in (2.1) by

$$
K_{n} u(x)=\sum_{(i, j) \in \mathcal{T}} \omega_{j} l\left(x, x_{i j}\right) u\left(x_{i j}\right) h_{i}, \quad x \in I,
$$

where $l(x, y):=y^{-1} \kappa(x / y)$. The Nyström solution $u_{n}(x)$ to the integral equation (1.1) is now defined by

$$
\left(1-K_{n}\right) u_{n}(x)=f(x), \quad x \in I .
$$

Note that (5.5) is a linear system in the values $u_{n}\left(x_{i j}\right),(i, j) \in \mathcal{T}$, and then $f(x)+K_{n} u_{n}(x)$ may be computed giving $u_{n}(x)$ for all $x \in I$.

To derive a stability result for (5.5), we need the following technical lemmas.

Lemma 5.1. If $v \in W_{1}^{k+1}(I)$ and $m \geq k$, then

$$
\left|\int_{I} v d x-\sum_{j=0}^{m} \omega_{j}^{(m)} v\left(\xi_{j}^{(m)}\right)\right| \leq c^{k+1}(1+m)^{-k}|| D^{k+1} v ; L_{1}(I) \|,
$$

where $c$ is independent of $v, k$ and $m$.

Proof. For any $\varphi \in \mathbf{P}_{m}$, the left-hand side of (5.6) can be estimated by

$$
\begin{aligned}
& \left|\int_{I}(v-\varphi) d x\right|+\left|\sum_{j=0}^{m} \omega_{j}^{(m)}(v-\varphi)\left(\xi_{j}^{(m)}\right)\right| \\
& \quad \leq\left\|v-\varphi ; L_{1}(I)\right\|+\sum_{j=0}^{m} \omega_{j}^{(m)}\left\|v-\varphi ; L_{\infty}(I)\right\| \leq c\left\|v-\varphi ; W_{1}^{1}(I)\right\| .
\end{aligned}
$$


Here we have used (5.2) and Sobolev's embedding theorem. Now the assertion follows from Proposition 3.1 (ii).

Lemma 5.2. Under the condition $\left(\mathrm{A} 3_{2}\right)$, we have

$$
\left\|\left(K \pi_{n}-K_{n}\right) u ; L_{q}(I)\right\| \leq c n^{-1}\left\|u ; L_{q, 2}(I)\right\|, \quad u \in L_{q, 2}(I), n \in \mathbf{N},
$$

where $c$ does not depend on $u$ and $n$ and $\pi_{n}$ denotes the truncation operator (4.4).

Proof. Applying Lemma 5.1 (with $k=1$ ) and the scaling argument, we obtain for any $u \in L_{q, 2}(I), n \in \mathbf{N}$ and $i \geq 2$

$$
\begin{aligned}
\mid \int_{I_{i}} l(x, y) u(y) d y & -\sum_{j=0}^{[\mu n]} \omega_{j} l\left(x, x_{i j}\right) u\left(x_{i j}\right) h_{i} \mid \\
& \leq c_{1}(1+[\mu n])^{-1} h_{i}^{2}\left\|D_{y}^{2} l(x, y) u(y) ; L_{1}\left(I_{i}\right)\right\| \\
& \leq c_{2} n^{-1}\left\|y^{2} D_{y}^{2} l(x, y) u(y) ; L_{1}\left(I_{i}\right)\right\| .
\end{aligned}
$$

Furthermore, the relation

$$
y^{2} D_{y}^{2} l(x, y) u(y)=\sum_{0 \leq r \leq 2} l_{r}(x, y) y^{r} D^{r} u(y)
$$

holds, where $l_{r}(x, y)=y^{-1} \kappa_{r}(x / y)$ and the functions $\kappa_{r}$ satisfy the first condition of (A1). From (5.7) we obtain for all $x \in I$ and $n$

$$
\left|\left(K \pi_{n}-K_{n}\right) u(x)\right| \leq c_{2} n^{-1} \sum_{0 \leq r \leq 2} \int_{0}^{1}\left|l_{r}(x, y)\right|\left|y^{r} D^{r} u\right| d y
$$

which implies the result by passing to $L_{q}$ norms.

We are now in a position to prove stability of the Nyström method in the weighted Sobolev spaces $L_{q, 2}(I)$.

Theorem 5.3. Assume (A1) and $\left(\mathrm{A} 3_{4}\right)$. Then the Nyström method (5.5) is stable in $L_{q, 2}(I)$, i.e.,

$$
\left\|\left(1-K_{n}\right) u ; L_{q, 2}(I)\right\| \geq c\left\|u ; L_{q, 2}(I)\right\|, \quad u \in L_{q, 2}(I), n \geq n_{0},
$$


where $n_{0}$ is sufficiently large and $c$ does not depend on $u$ and $n$.

Proof. Step 1. First we verify that the operators $1-K \pi_{n}$ are stable in $L_{q, 2}(I)$. Lemma 4.2 implies the stability of these operators in $L_{q}(I)$ since one has the matrix representation

$$
1-K \pi_{n}=\left(\begin{array}{cc}
\pi_{n}(1-K) \pi_{n} & 0 \\
-\pi_{n} K\left(1-\pi_{n}\right) & 1
\end{array}\right)
$$

with respect to the direct sum $L_{q}(I)=\pi_{n}\left(L_{q}(I)\right) \oplus\left(1-\pi_{n}\right)\left(L_{q}(I)\right)$. Moreover, for $j=1,2$,

$$
\left\|x^{j} D^{j} u ; L_{q}(I)\right\| \leq\left\|x^{j} D^{j}\left(1-K \pi_{n}\right) u ; L_{q}(I)\right\|+\left\|x^{j} D^{j} K \pi_{n} u ; L_{q}(I)\right\|
$$

and by $\left(\mathrm{A}_{2}\right)$ and the stability of $1-K \pi_{n}$ in $L_{q}(I)$

$$
\begin{aligned}
\left\|x^{j} D^{j} K \pi_{n} u ; L_{q}(I)\right\| & \leq c\left\|\pi_{n} u ; L_{q}(I)\right\| \leq c\left\|u ; L_{q}(I)\right\| \\
& \leq c_{1}\left\|\left(1-K \pi_{n}\right) u ; L_{q}(I)\right\| .
\end{aligned}
$$

These estimates obviously yield (5.8) with $K \pi_{n}$ in place of $K_{n}$.

Step 2. We show that for any $n \in \mathbf{N}, u \in L_{q, 2}(I)$ and $j=0,1,2$, the estimate

$$
\left\|x^{j} D^{j}\left(K \pi_{n}-K_{n}\right) u ; L_{q}(I)\right\| \leq c n^{-1}\left\|u ; L_{q, 2}(I)\right\|
$$

holds. Then the stability of (5.5) follows from that of $1-K \pi_{n}$ by small perturbation with respect to the $L_{q, 2}$ operator norm. Note that Lemma 5.2 implies (5.9) if $j=0$. For $j=1,2$, we use the fact that $K^{(j)}=x^{j} D^{j} K$ is the Mellin convolution operator with kernel $x^{j} D^{k} \kappa$ and that $K_{n}^{(j)}=x^{j} D^{j} K_{n}$ is the approximate operator (5.4) corresponding to $K^{(j)}$. Since, by $\left(\mathrm{A} 3_{4}\right), K^{(j)}$ satisfies condition $\left(\mathrm{A} 3_{2}\right)$, it suffices to apply Lemma 5.2 to those operators.

Remark 5.4. Using the more traditional approach of showing that $\left\|\left(K \pi_{n}-K_{n}\right) K_{n}\right\|_{\infty} \rightarrow 0$ as $n \rightarrow \infty$, one can also prove the stability of (5.5) in $L_{\infty}(I)$; cf. [9] in the case of the $h$-version of the Nyström method.

We are now ready to state our result on exponential convergence of the Nyström method. 
Theorem 5.5. Assume $f \in B_{q}^{\varrho}(I), \varrho>0,(\mathrm{~A} 1),\left(\mathrm{A} 2^{\varrho}\right),\left(\mathrm{A} 4^{\varrho}\right)$ and $\left(\mathrm{A} 5^{\circ}\right)$. Then the Nyström method converges with the error bound (4.3) where $u_{n}$ denotes the solution of (5.5).

Proof. Using the relation $\left(1-K_{n}\right) u_{n}=(1-K) u$ and Theorem 5.3, we obtain

$$
\begin{aligned}
\left\|u-u_{n} ; L_{q, 2}(I)\right\| & \leq c\left\|\left(1-K_{n}\right)\left(u-u_{n}\right) ; L_{q, 2}(I)\right\| \\
& =c\left\|\left(K-K_{n}\right) u ; L_{q, 2}(I)\right\| .
\end{aligned}
$$

Recall that condition $\left(\mathrm{A} 5^{\circ}\right)$ is stronger than $\left(\mathrm{A} 3_{4}\right)$. Furthermore, by assumption and Theorem 2.1, we have $u \in B_{q}^{\varrho}(I)$ so that it remains to apply Lemma 5.7 below to the operators $x^{j} D^{j}\left(K-K_{n}\right), j=0,1,2$,

口

Remark 5.6. Under the conditions of the preceding theorem, we obtain the exponential convergence rate

$$
\left\|u-u_{n} ; L_{q, j}(I)\right\| \leq c e^{-b n}, \quad c, b \text { independent of } n,
$$

for any $j \in \mathbf{N}$. Indeed, if we apply the operators $x^{j} D^{j}$ to the relation

$$
u-u_{n}=K u-K_{n} u_{n}=\left(K-K_{n}\right) u+K_{n}\left(u-u_{n}\right),
$$

we obtain with the notation in Step 2 of the proof of Theorem 5.3

$$
x^{j} D^{j}\left(u-u_{n}\right)=\left(K^{(j)}-K_{n}^{(j)}\right) u+K_{n}^{(j)}\left(u-u_{n}\right),
$$

where the kernel of $K^{(j)}$ also satisfies the assumption $\left(\mathrm{A} 5^{\circ}\right)$. Lemma 5.7 below yields the exponential rate for the first term in (5.10). For the second term, this is a consequence of Theorem 5.5 and the uniform boundedness of $K_{n}^{(j)}$, the latter following from Lemma 5.2 applied to $K^{(j)}$ and the continuity of $K^{(j)}: L_{2}(I) \rightarrow L_{2, k}(I)$ for any $j$ and $k$.

Lemma 5.7. If the assumptions $u \in B_{q}^{\varrho}(I), \varrho>0$ and $\left(\mathrm{A} 5^{\circ}\right)$ are fulfilled, then

$$
\left\|\left(K-K_{n}\right) u ; L_{q}(I)\right\| \leq c e^{-b n}
$$


where the constants $c$ and $b$ do not depend on $n$.

Proof. We proceed similarly as in the proof of Theorem 3.4. First we have

$$
\begin{aligned}
\left\|K\left(1-\pi_{n}\right) u ; L_{q}(I)\right\| & \leq c\left\|\left(1-\pi_{n}\right) u ; L_{q}(I)\right\| \\
& \leq c \sigma^{(n-1) \varrho}\left\|x^{-\varrho} u ; L_{q}(I)\right\| \leq c \sigma^{n \varrho} .
\end{aligned}
$$

Here and in the sequel $c$ and $b$ denote various constants which are independent of $n$. It remains to show the estimate

$$
\left\|\left(K \pi_{n}-K_{n}\right) u ; L_{q}(I)\right\| \leq c \sigma^{b n} .
$$

Applying Lemma 5.1 (with $k=[\nu n], m=[\mu n]$ ) and the scaling argument, we obtain for all $i \geq 2$ and $\nu \in(0, \mu)$

$$
\begin{aligned}
& \left|\int_{I_{i}} l(x, y) u(y) d y-\sum_{j=0}^{[\mu n]} \omega_{j} l\left(x, x_{i j}\right) u\left(x_{i j}\right) h_{i}\right| \\
& \leq c\{c /(1+[\mu n])\}^{[\nu n]} h_{i}^{1+[\nu n]}\left\|D_{y}^{1+[\nu n]} l(x, y) u(y) ; L_{1}\left(I_{i}\right)\right\| \\
& \leq c\{c / 1+[\mu n])\}^{[\nu n]} x_{i}^{\varrho}\left\|y^{1+[\nu n]-\varrho} D_{y}^{1+[\nu n]} l(x, y) u(y) ; L_{1}\left(I_{i}\right)\right\| .
\end{aligned}
$$

Define the functions $\kappa_{j}$ and $l_{j}$ by

$$
y^{-1} \kappa_{j}(x / y)=l_{j}(x, y)=y^{j} D_{y}^{j} l(x, y) .
$$

Recall that $y^{-1} \kappa(x / y)=l(x, y)$. Then

$$
\int_{0}^{\infty} x^{1 / q-1}\left|\kappa_{j}(x)\right| d x \leq d^{j+1} j !, \quad j=0,1, \ldots,
$$

where $d$ is independent of $j$. This follows easily from condition $\left(\mathrm{A} 5^{\circ}\right)$, using Lemma 2.2 and the relations $(x D)^{j}=(D x-1)^{j}$,

$$
\left(D_{y} y\right)^{j} l(x, y)=(-1)^{j} y^{-1}\left((x D)^{j} \kappa\right)(x / y) .
$$

Now let $K_{j}$ be the Mellin convolution operator with kernel function

$$
\{c /(1+[\mu n])\}^{\{\nu n]}\left|\kappa_{j}\right|(x),
$$


and define the functions $v_{j}$ by

$$
v_{j}=0 \quad \text { on } I_{1}, \quad v_{j}(x)=x_{i}^{\varrho}\left|x^{j-\varrho} D^{j} u(x)\right| \quad \text { on } I_{i}, i \geq 2 .
$$

Since $u \in B_{q}^{\varrho}(I)$, we then have

$$
\left\|v_{j} ; L_{q}(I)\right\| \leq d^{j+1} j !, \quad j=0,1, \ldots .
$$

Furthermore, with the notation of (5.14)

$$
y^{m-\varrho} D_{y}^{m} l(x, y) u(y)=\sum_{j=0}^{m}\left(\begin{array}{c}
m \\
j
\end{array}\right) l_{j}(x, y) y^{m-j-\varrho} D^{m-j} u(y)
$$

for any $m$, and combining the estimates (5.13) we can write

$$
\left|\left(K \pi_{n}-K_{n}\right) u(x)\right| \leq \sum_{j=0}^{1+[\nu n]}\left(\begin{array}{c}
1+[\nu n] \\
j
\end{array}\right) K_{j} v_{1+[\nu n]-j}(x), \quad x \in I .
$$

Using (5.15) and (5.16), this implies

(5.17) $\left\|\left(K \pi_{n}-K_{n}\right) u ; L_{q}(I)\right\|$

$$
\begin{aligned}
& \leq \sum_{j=0}^{1+[\nu n]} c\left\{\frac{c}{1+[\mu n]}\right\}^{[\nu n]}\left(\begin{array}{c}
1+[\nu n] \\
j
\end{array}\right) d^{j+1} j ! d^{2+[\nu n]-j}(1+[\nu n]-j) ! \\
& \leq c\{c /(1+[\mu n])\}^{\{\nu n]}(2+[\nu n]) ! \leq c\{c(1+[\nu n]) /(1+[\mu n])\}^{[\nu n]} .
\end{aligned}
$$

Here we have used the estimate

$$
(2+[\nu n]) ! /(1+[\nu n])^{[\nu n]} \leq 2^{1+[\nu n]} .
$$

As in the proof of Theorem 3.4, the last quantity in (5.17) can be estimated by $c \sigma^{b n}$ if $\nu \in(0, \mu)$ is chosen sufficiently small. This completes the proof of (5.12).

Remark 5.8. All results of this section extend with the same proofs to the case where the collocation points $x_{i j}$ are defined by means of the Gauss-Legendre nodes $\xi_{j}^{(m)}$ on $I$, since the corresponding weights $\omega_{j}^{(m)}$ satisfy condition (5.2) again. 
Finally we note that the results on the Nyström method remain valid if we choose a composite quadrature rule based on a linear degree distribution on the mesh $\Delta_{n, \sigma}$ (i.e., a suitable interpolatory projection $Q_{n}$ onto $\mathcal{S}_{n, \sigma, \mu}^{\text {lin }}$ is taken in (5.3)) and if $\mu$ is sufficiently large.

Acknowledgment. The author is grateful to the referees for valuable suggestions.

\section{REFERENCES}

1. P.M. Anselone, I.H. Sloan, Integral equations on the half-line, J. Integral Equations 9 (Suppl.) (1985), 3-23.

2. I. Babuška, B.Q. Guo and E.P. Stephan, On the exponential convergence of the $h$-p-version for boundary element Galerkin methods on polygons, Math. Methods Appl. Sci. 12 (1990), 413-427.

3. I. Babuška and M. Suri, The $p$ - and h-p-versions of the finite element method. An overview, Tech. Note BN-1101, University of Maryland, College Park, 1989.

4. G.A. Chandler and I.G. Graham, Product integration-collocation methods for noncompact integral operator equations, Math. Comp. 50 (1988), 125-138.

5. J. Elschner, Asymptotics of solutions to pseudodifferential equations of Mellin type, Math. Nachr. 130 (1987), 267-305.

6. - On spline collocation for convolution equations, Integral Equations Operator Theory 12 (1989), 486-510.

7. - On spline approximation for a class of noncompact integral equations, Math. Nachr. 146 (1990), 271-321.

8. I. Gohberg and I.A. Feldman, Convolution equations and projection methods for their solution, Amer. Math. Soc. Transl. 41, Providence, 1974.

9. I.G. Graham and G.A. Chandler, High order methods for linear functionals of solutions of second kind integral equations, SIAM J. Numer. Anal. 25 (1988), $1118-1137$.

10. B. Guo and I. Babuška, The $h-p$-version of the finite element method. Part I: The basic approximation results. Part 2: General results and applications, Comput. Mechanics 1 (1986), 21-41, 203-226.

11. P.-J. Laurent, Approximation et optimisation, Hermann, Paris, 1972.

12. I.P. Natanson, Konstruktive Funktionentheorie, Akademie-Verlag, Berlin, 1955.

13. S. Prößdorf and B. Silbermann, Numerical analysis for integral and related operator equations, Akademie-Verlag, Berlin, 1991.

14. L.L. Schumaker, Spline functions: Basic theory, Wiley, New York, 1981.

15. E.P. Stephan and M. Suri, The h-p-version of the boundary element method on polygonal domains with quasiuniform meshes, Math. Model. Numer. Anal. 25 (1991), 783-807. 
16. G. Szegö, Orthogonal polynomials, Amer. Math. Soc., New York, 1959.

Institut für Angewandte Analysis und Stochastik, Hausvogteiplatz 5-7, D-0-1086 Berlin, Germany 